\title{
Characteristics of Optical and Photoconductive Properties in Bulk and Thin Film of $\mathrm{TlS}_{2}$ Single Crystals
}

\author{
A.M. BADR \\ Physics Department, Faculty of Science, South Valley University \\ Aswan, Egypt
}

(Received April 2, 2007; revised version July 17, 2007)

\begin{abstract}
The photoconductivity measurements were carried out for bulk single crystals of $\mathrm{TlS}_{2}$ by using the steady state (dc) method in order to elucidate the nature of the dc photoconductivity in these crystals. The photoconductivity measurements were carried out in the temperature range of 77-300 K, excitation intensity range of 2150-5050 lx, applied voltage range of 10-25 V, and wavelength range of $400-915 \mathrm{~nm}$. As a result of the dc photoconductivity, the temperature dependence of the energy gap width was described and the temperature coefficient of the band gap was determined. Reflectance and transmittance spectra of the $\mathrm{TlS}_{2}$ thin films were measured in the incident photon energy range of $2.1-2.45 \mathrm{eV}$ and in the temperature range of 77-300 K. With the aid of these spectra, the temperature dependence of optical transports and parameters were elucidated. In the low energy region of the studied incident photon energy range, the above-mentioned spectra were analyzed for describing the refractive index as a function of wavelength. As results of the refractive index-wavelength variations, both the oscillator and dispersion energies of the refractive index were thereafter estimated.
\end{abstract}

PACS numbers: 71.35.Cc, 73.50.Gr, 73.50.Pz, 78.20.-e, 78.20.Ci

\section{Introduction}

The chalcogenide semiconductor compounds (of the $\mathrm{A}^{\mathrm{III}} \mathrm{B}^{\mathrm{VI}}$ group) are of interest because investigations of their physical properties open up the possibility of important applications in technology [1]. According to our best knowledge, the Tl-S modifications (e.g., TlS, $\mathrm{Tl}_{2} \mathrm{~S}, \mathrm{Tl}_{4} \mathrm{~S}_{3}$, or $\mathrm{TlS}_{2}$ crystals) are unique semiconducting materials, but they are the less studied compounds in the $\mathrm{A}^{\mathrm{III}} \mathrm{B}^{\mathrm{VI}}$ group. Due to difficulties in obtaining the above-mentioned modifications in their single crystal forms, however, little is known about their physical properties [2]. Although 
TIS single crystal is known as a good photoconductor, its electronic structure is not well known [3]. Synthetic $\mathrm{Tl}_{2} \mathrm{~S}$ is known to be a black, soft, and extremely laminar substance. In nature it was described as the rare mineral carlinite [4]. Earlier crystal structure work $[5,6]$ has shown that the atomic arrangement is similar to that of the anti-CdI $\mathrm{C}_{2}$ type, but with lattice parameters $a$ and $c$ tripled. A new description and a new discussion of the stereochemistry have been investigated by Giester et al. [7], in which they reported that the interatomic distances will always be given to two decimals $(\sigma<0.01 \AA)$, although for the $\mathrm{Tl}-\mathrm{Tl}$ distances three decimals would be adequate $(\sigma<0.005 \AA)$. Photoemission studies, using a synchrotron photon source, on TIS crystals have been reported by Kashida et al. [3]. They reported that the binding energies of the $\mathrm{Tl} 5 d_{5 / 2}$ and $\mathrm{Tl} 5 d_{3 / 2}$ core levels for $\mathrm{Tl}_{2} \mathrm{~S}$ crystal are 15.55 and $13.36 \mathrm{eV}$, respectively. The electrical properties of $\mathrm{Tl}_{2} \mathrm{~S}$ and TIS single crystals have also been reported in this investigation, the same studies for $\mathrm{Tl}_{4} \mathrm{~S}_{3}$ have been recorded in our paper [8]. The optical absorption studies of $\mathrm{Tl}_{2} \mathrm{~S}$ have been investigated by Estrella et al. [9] and suggest that there are direct forbidden transitions with a band gap of $1.12 \mathrm{eV}$. Characteristics of photoconductivity in $\mathrm{Tl}_{2} \mathrm{~S}$ layered single crystals have been reported in our paper [10], and the same studies for $\mathrm{Tl}_{4} \mathrm{~S}_{3}$ have been reported in our paper [11].

In this work, our aim is to elucidate the photoconductive properties in bulk samples of $\mathrm{TlS}_{2}$ single crystals and optical properties for thin films evaporated from the above-mentioned crystals. These investigations were carried out as a part of a work plan for covering the physical properties of the above-mentioned modification $\mathrm{TlS}_{2}$, and because of: (1) the $\mathrm{TlS}_{2}$ is the less studied modification of the Tl-S semiconducting system; (2) the experimental measurements showed that the obtained $\mathrm{TlS}_{2}$ single crystals are good photoconductors and have high photosensitivity; and (3) due to difficulties in obtaining $\mathrm{TlS}_{2}$ single crystals of the modification mentioned, there is no enough information in the literature about its physical properties.

\section{Experimental details}

A special technique was used for growing single crystals of the $\mathrm{TlS}_{2}$ compound. Pure $(5 \mathrm{~N})$ elements (Aldrich) were used as starting materials of the compound under investigation. Starting materials were placed in an evacuated $\left(10^{-6}\right.$ Torr $)$ silica tube. Thereafter, the silica tube was fixed in a suitable set inside the furnace tube of the Lenton thermal design (LTD) technique [8]. Crystals of $\mathrm{TlS}_{2}$ were obtained via cooling rate of $3^{\circ} \mathrm{C} / \mathrm{h}$. The selected set within the furnace tube was exposed to a temperature that is high enough to the melt of the compound under investigation. During this stage, the silica tube was gently shaken for intermixing the elements of the compound, then the silica tube was kept for $24 \mathrm{~h}$ within the selected set of the furnace tube. The solidification diagram of the $\mathrm{Tl}-\mathrm{S}$ system showed that $\mathrm{TlS}_{2}$ compound can be formed at $125^{\circ} \mathrm{C}$ [12]. It is evident that this program is short, but it needs a month before switching off the 


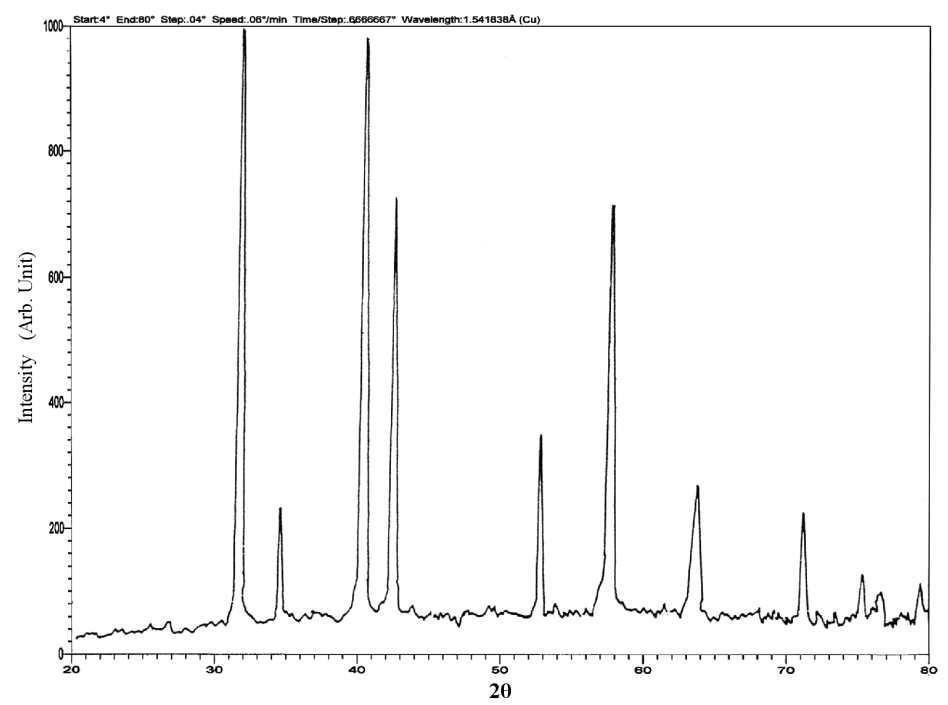

Fig. 1. The X-ray diffraction pattern of the samples under investigation.

furnace. The prepared crystals were analyzed by X-ray diffraction. Consequently, it was evident from the comparison between the recorded (see Fig. 1) and standard X-ray diffraction patterns [13] that the obtained samples were prepared in their monocrystal form. In line with this comparison, the obtained crystals were found with tetragonal symmetry, space group: $P 4_{2} / n m c$ (137) and lattice parameters: $a=23.17 \AA, c=54.76 \AA$, and $Z=384$. The bulk sample, which was used in the photoconductivity (PC) measurements, was prepared by splitting the obtained crystals. This led to the situation that the resultant surfaces of the prepared bulk samples were mirror-like without any mechanical treatment. Then the sample used in measurements was mounted on the cold finger inside a cryostat (Oxford DN1704-type), which was evacuated to about $10^{-4}$ Torr. The temperature inside the cryostat was controlled by a digital temperature controller (Oxford ITC601type). The contacts between the samples and the metal electrodes were made by using silver paste. The electrical contacts have been tested to analyze their $I-V$ characteristics and are found to be ohmic in the used range of the applied voltage. Excitation was done by a tungsten lamp of $1000 \mathrm{~W}$, which is connected to a variac for adjusting the light intensity at the sample surface. The adjustment was carried out for the longest distance between the light source and the sample with which enough clear spectral distribution can be obtained. In measuring the light intensity dependence of the photocurrent, the incident light intensity has been varied by dividing the above-mentioned longest distance into a number of smaller distances. The incident light was focused by using an optical system consisting of two convex lenses, which enables a homogeneous illumination to be obtained. The dc PC measurements were made with the aid of an electrometer (Keithly 610-type) 
and a monochromator (Carl Zeiss M4GII-type). A thermal evaporation technique was used for obtaining the desired thickness of $\mathrm{TlS}_{2}$ thin films with which the obtained films were obtained transparent in appearance and brown in color. A molybdenum boat was charged with the starting materials in granular form and the vacuum chamber was pumped down to about $\approx 10^{-6}$ Torr. Then the boat was gradually heated until the material under work was started to be evaporated onto glass cleaned substrates. The thermal evaporation processes were performed by using a coating unit Edwards Coating System of E306A-Mark including a thermal evaporation chamber. The evaporation rate was slow, where higher evaporation rates led to non-uniform film and low sticking. It was found that films up to $60 \mathrm{~nm}$ were completely transparent and become opaque for higher thicknesses. The width and length of the resultant films were used in addition to their masses and densities for determining the thicknesses of the obtained films. The thickness of the films under investigation was calculated to be of about $60 \mathrm{~nm}$. Also a UV-visible double beam spectrophotometer of model UV-1650PC was employed to record the reflectance and transmittance spectra.

\section{Results and discussion}

\subsection{Photoconductive properties}

The dc PC measurements of $\mathrm{TlS}_{2}$ bulk single crystals were carried out in a wide range of the incident photon energy. The crystals under investigation were found as good photoconductors and have a high photosensitivity. Spectral distributions of the photocurrent for bulk samples of $\mathrm{TlS}_{2}$ single crystals were studied in the wavelength range of 400-915 nm at different values of temperature, different values of applied voltage, and different values of light intensity. Photocurrent was evaluated by subtracting the darkness value of the current from the values under illumination. The dependence of the $\mathrm{dc} \mathrm{PC}$ spectra on temperature in the range of $77-300 \mathrm{~K}$ is depicted in Fig. 2. A voltage of $10 \mathrm{~V}$ was applied in this experiment, and a light intensity of $2150 \mathrm{~lx}$ was used for excitation process. In view of this figure, the highest value of the photocurrent (photopeak) increases with increasing temperature, which may be due to the trapping (capture) processes. Also the dc PC spectra have one photopeak in the whole studied range of temperature, which is associated with direct band-band transition. The photopeak was found for $2.43 \mathrm{eV}$ at $77 \mathrm{~K}$ and shifts toward low photon energy with increasing temperature and at $300 \mathrm{~K}$ the photopeak was found at $2.047 \mathrm{eV}$. For an intrinsic semiconductor, a spectral sensitivity curve can be obtained using the following relation [14]:

$$
S(E)=\frac{1}{1+\exp \left(B\left(E_{0}-E\right)\right)},
$$

where $B$ is constant, $E$ is the energy at any wavelength, and $E_{0}$ is the energy at the threshold wavelength. The $S(E)-E$ curve has a flat response at short wavelength and an exponential fall with energy at long wavelength. Variation of the 


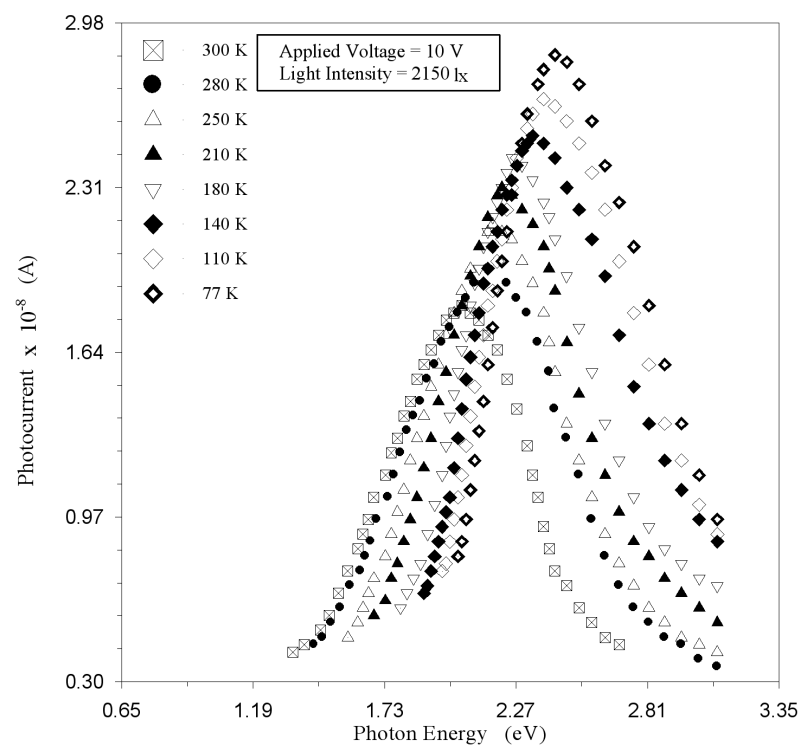

Fig. 2. The spectral distribution of the dc $\mathrm{PC}$ for a bulk sample of $\mathrm{TlS}_{2}$ single crystal in the temperature range $77-300 \mathrm{~K}$.

sensitivity is assumed to be resulted due to the distribution of the energy levels, where photoelectrons originate from or to these levels. Busch [15] and Rose [16] have considered such a distribution of centers. Assuming that $N(E) \mathrm{d} E$ represents the number of levels per unit volume lying between $E$ and $E+\mathrm{d} E$ and if the material is irradiated by a given quantum energy $E_{\lambda}$, then the amount of absorption and hence the sensitivity will be proportional to the total number of centers of energy lower than $E_{\lambda}$, i.e.

$$
S(E) \approx \int_{0}^{E_{\lambda}} N(E) \mathrm{d} E,
$$

which leads to

$$
N(E)=G \frac{\mathrm{d} S(E)}{\mathrm{d} E},
$$

where $G$ is constant. Using Eq. (1) in Eq. (2), we get

$$
N(E)=\left[G B \mathrm{e}^{B\left(E_{0}-E\right)}\right] /\left[1+\mathrm{e}^{B\left(E_{0}-E\right)}\right]^{2} .
$$

In view of the above expression, the number of electrons that will be present in the conduction band due to thermal excitation from level $E$ can be calculated from the relation

$$
n=C(G / B)^{1 / 2} \frac{\pi}{\sin (\pi a)} \exp \left(-\left(E_{0} / 2 k T\right)\right),
$$

where $C$ is a constant and $G$ means the photoconductivity gain, which is defined as the photosensitivity of a photoconductor in terms of the number of charge car- 
riers (the charge carriers pass between the electrodes per second for each photon absorbed). This definition has been reported in Ref. [17]. The above relation is similar to that obtained for the Hall effect using a simple theory of electrons excited into the conduction band from one definite energy level [18]:

$$
n=n_{0} \exp \left(-\frac{E_{\mathrm{g}}}{2 k T}\right),
$$

where $E_{\mathrm{g}}$ is the intrinsic forbidden gap. Therefore, the comparison of Eq. (4) with Eq. (5) leads to $E_{\mathrm{g}}=E_{0}$ for intrinsic conductivity. So when $E=E_{\mathrm{g}}$, the sensitivity $S$ can be described by the following relation:

$$
S=\frac{1}{1+\exp \left(E_{0}-E_{\mathrm{g}}\right)}=\frac{1}{2} .
$$

This leads to an important conclusion that the activation energy is determined from the spectral curve as the point, where the sensitivity has fallen to half of its value that means

$$
E_{0}=E_{\mathrm{g}}=\text { quantum energy at } \lambda_{1 / 2} .
$$

Therefore, the forbidden gap $E_{\mathrm{g}}$ can be determined by estimating quantum energy at $\lambda_{1 / 2}$. On the other hand, $E_{\mathrm{g}}=h \nu=h c / \lambda_{1 / 2}$.

With the aid of the $\lambda_{1 / 2}$ technique, the energy gap $E_{\mathrm{g}}$ values were calculated over the whole range of temperature under investigation that were obtained as a result of the corresponding spectra of the dc PC spectra. Plotting the estimated values of the band gap width versus the corresponding temperatures (see Fig. 3), it was found that the energy gap of the $\mathrm{TlS}_{2}$ bulk single crystal decreases as temperature increases. Using Fig. 3, the value of $E_{\mathrm{g}}$ was calculated to be $\approx 1.65 \mathrm{eV}$

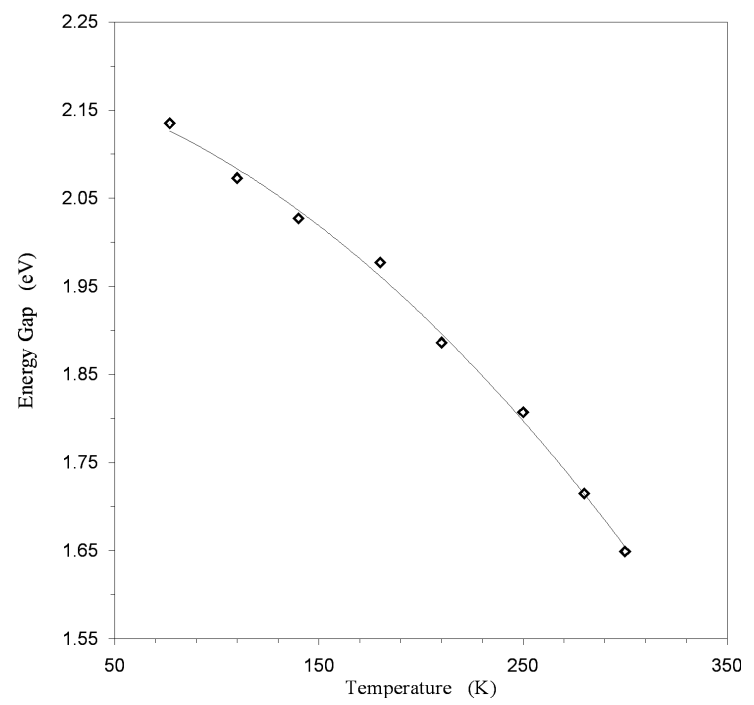

Fig. 3. The energy gap versus temperature plotted for a bulk sample of $\mathrm{TlS}_{2}$ single crystal as a result of the dc $\mathrm{PC}$ data. 
at room temperature, and increased with decreasing temperature to be $\approx 2.14 \mathrm{eV}$ at $77 \mathrm{~K}$. The values of $E_{\mathrm{g}}$ were evaluated as results of the temperature dependence of the $\mathrm{dc}$ PC. The temperature coefficient $\left(\mathrm{d} E_{\mathrm{g}} / \mathrm{d} T\right)$ can be determined by using the temperature dependence of the energy gap $\left(E_{\mathrm{g}}\right.$ versus $\left.T\right)$. It can be calculated using the linear fitting of the plot illustrated in Fig. 3. The temperature coefficient was calculated to be $\approx-2.65 \times 10^{-3} \mathrm{eV} / \mathrm{K}$. According to Ref. [19], Fig. 3 suggests that the contribution of the electron-phonon interaction may be larger than that of the lattice expansion, where both the contributions of electron-phonon interaction term and the lattice expansion term can be described using the following relation:

$$
\left(\frac{\mathrm{d} E_{\mathrm{g}}}{\mathrm{d} T}\right)=\left(\frac{\mathrm{d} E_{\mathrm{g}}}{\mathrm{d} T}\right)_{\mathrm{L}-\mathrm{Ex}}+\left(\frac{\mathrm{d} E_{\mathrm{g}}}{\mathrm{d} T}\right)_{\mathrm{E}-\mathrm{P}},
$$

where the first term depends on the lattice expansion (L-Ex) and the second term depends on the electron-phonon (E-P) interactions.

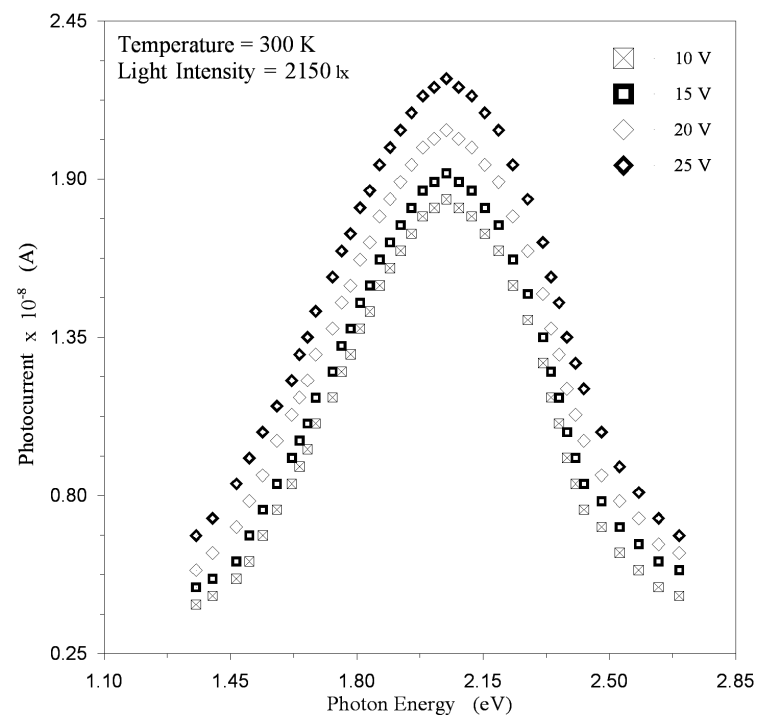

Fig. 4. The spectral distribution of the dc PC for a bulk sample of $\mathrm{TlS}_{2}$ single crystal at different values of the applied voltage.

The spectral distributions of the dc PC at different bias voltages and light intensities of the $\mathrm{TlS}_{2}$ bulk single crystal were carried in the wavelength range from 460 to $915 \mathrm{~nm}$. The above-mentioned spectral distributions are shown in Figs. 4 and 5. At different values of the applied voltage (from 10 to $25 \mathrm{~V}$ ), the dc PC spectra of the bulk samples were measured with fixed values of both temperature $300 \mathrm{~K}$ and light intensity $2150 \mathrm{~lx}$. The dc PC spectra at different light intensities (from 2150 to $5050 \mathrm{~lx}$ ) were measured with fixed values of both temperature $300 \mathrm{~K}$ and applied voltage $10 \mathrm{~V}$. In view of Figs. 4 and 5, the shape of the spectral distribution characteristics is particularly found to be independent of both the 


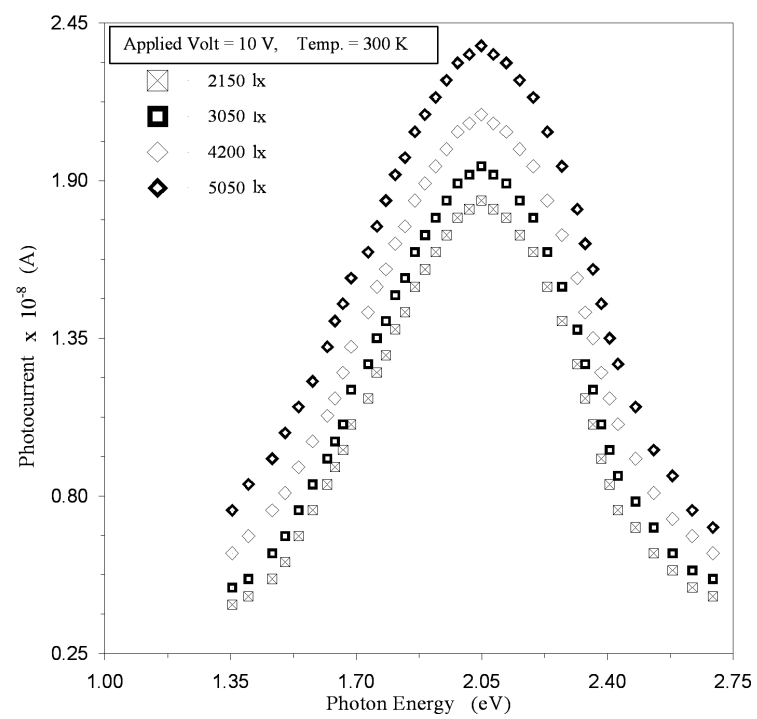

Fig. 5. The spectral distribution of the dc $\mathrm{PC}$ for a bulk sample of $\mathrm{TlS}_{2}$ single crystal at different values of the light intensity.

light intensity and the applied bias voltage where the number of excited carriers increases with increasing light intensity that makes the photocurrent stronger. It was observed that the photocurrent shifts toward its higher values as light intensity or applied bias voltage increases (see Figs. 4 and 5). Because of the relation between the carrier velocity $v$, the carrier mobility $\mu$ and the electric field $E, v$ can be simply written in the form $v=\mu E[17], v$ is proportional to the applied voltage $V$ ( $E$ is also proportional to $V$ ). So the light intensity generates the carriers to be drifted by increasing the applied voltage, leading to an increase in the carrier velocity.

\subsection{Optical properties}

The transmittance $(T)$ and reflectance $(R)$ spectra of the $\mathrm{TlS}_{2}$ thin films were measured in the temperature range of $77-300 \mathrm{~K}$. The recorded spectra were carried out in the incident photon energy range of 2.1-2.45 eV. In line with Figs. 6 and 7 , typical spectra of the transmittance and reflectance for the $\mathrm{TlS}_{2}$ thin films are observed from 77 to $300 \mathrm{~K}$. But these figures show that there is a shift in either transmittance curves or reflectance curves toward the lower values of the incident photon energy with the increase in temperature.

As it is known, optical measurements are productive tools for understanding the band structure, energy gap width, and optical parameters of both crystalline and amorphous non-metallic materials. The optical absorption coefficient $(\alpha)$ is related to the transmittance $(T)$ of a sample with thickness $(d)$ through the relation 


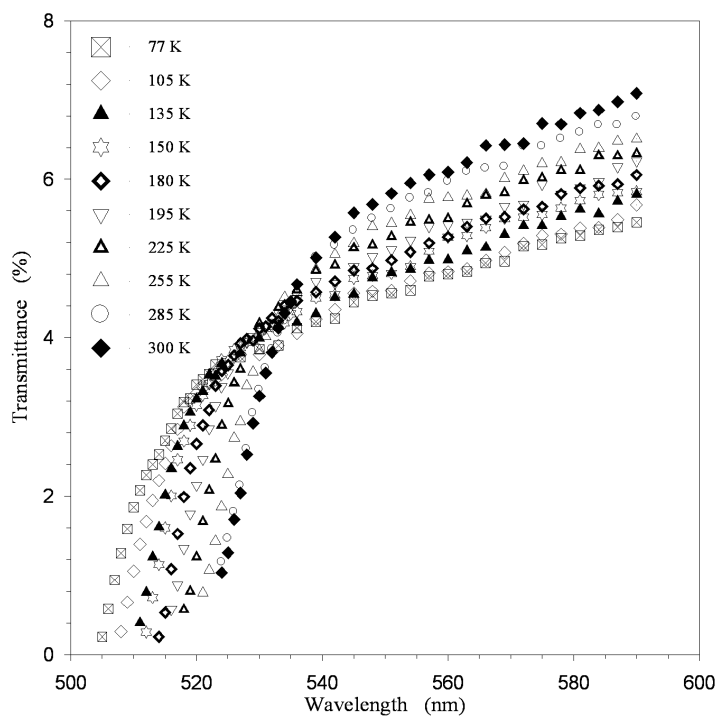

Fig. 6. The temperature dependence of transmittance spectra in $\mathrm{TlS}_{2}$ thin film.

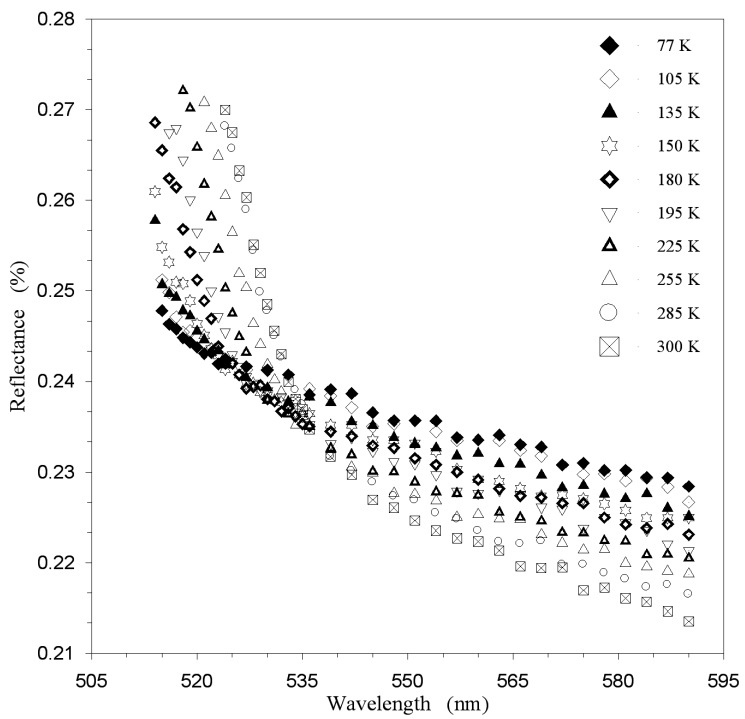

Fig. 7. The temperature dependence of reflectance spectra in $\mathrm{TlS}_{2}$ thin film.

$$
\alpha=\frac{1}{d} \ln \left(\frac{1}{T}\right) .
$$

This relation was used for calculating the values of the absorption coefficient (see Fig. 8). It is obvious from this figure that the absorption coefficient exhibits a long band tail at low energies. This weak absorption tail is most probably originating from defects and impurity states within the band gap. Tailing of the band states 


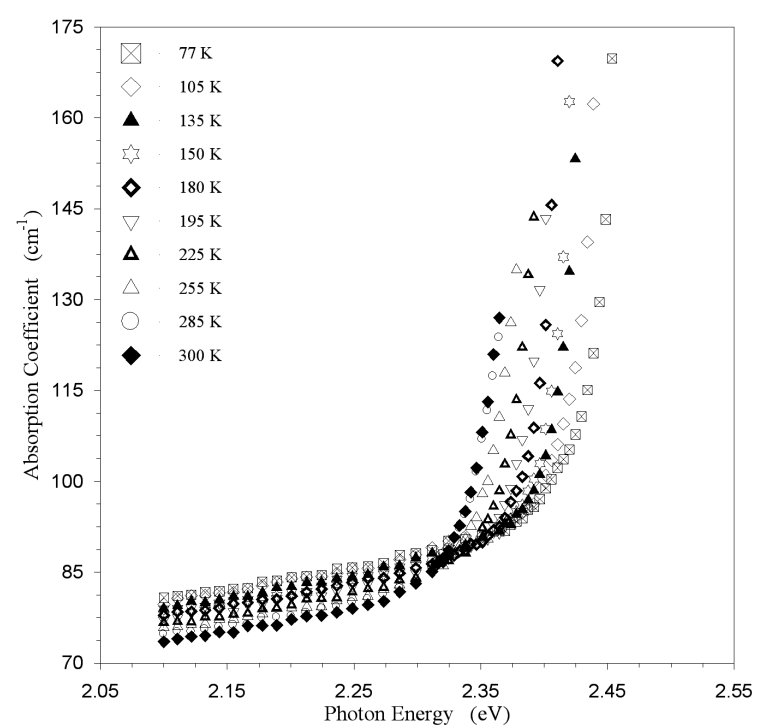

Fig. 8. The dependence of absorption coefficient on temperature for a thin film of $\mathrm{TlS}_{2}$.

into the gap width may be induced from a large concentration of free carriers resulting from the screened Coulomb interaction between carriers that perturbs the band edges. Thereafter it steeply increases with an increase in photon energy near the fundamental edge. In the region of the photon energy that has values greater than those of the exponential edge region, the absorption coefficient linearly increases with increasing the incident photon energy. These distinct regions are obviously depicted in Fig. 8. Also it is clear from this figure that the values of the absorption coefficient up to those of the exponential edge region (high absorption region or linear portion) undergo a shift toward low values of the photon energy with increasing temperature. Also the exponential edge exhibits a shift toward low values of the photon energy with an increase in temperature.

In the high absorption regions (a linear increase in $\alpha$ with an increase in incident photon energy), the relationship between the absorption coefficient and the incident photon energy $(h \nu)$ is governed by the relation [20, 21]:

$$
\alpha=\frac{\left[A\left(h \nu-E_{\mathrm{g}}\right)^{n}\right]}{h \nu},
$$

where $A$ is constant depending on the transition probability, $E_{\mathrm{g}}$ is the width of the band gap, and $n$ is an index that characterizes the optical absorption processes in the films under investigation. The analysis of experimental results showed that a proportionality is revealed between the absorption coefficient and the frequency of the photon energy in the form $\left(h \nu-E_{\mathrm{g}}\right)^{n}$. The exponent $n$ can take one of the four values: $2,1 / 2,3$, and $3 / 2$, which define the type of the optical transition. Theoretically $m$ is equal to $2,1 / 2,3$, or $3 / 2$ for the indirect allowed, direct allowed, indirect forbidden, and direct forbidden transitions, respectively [22]. For the case 


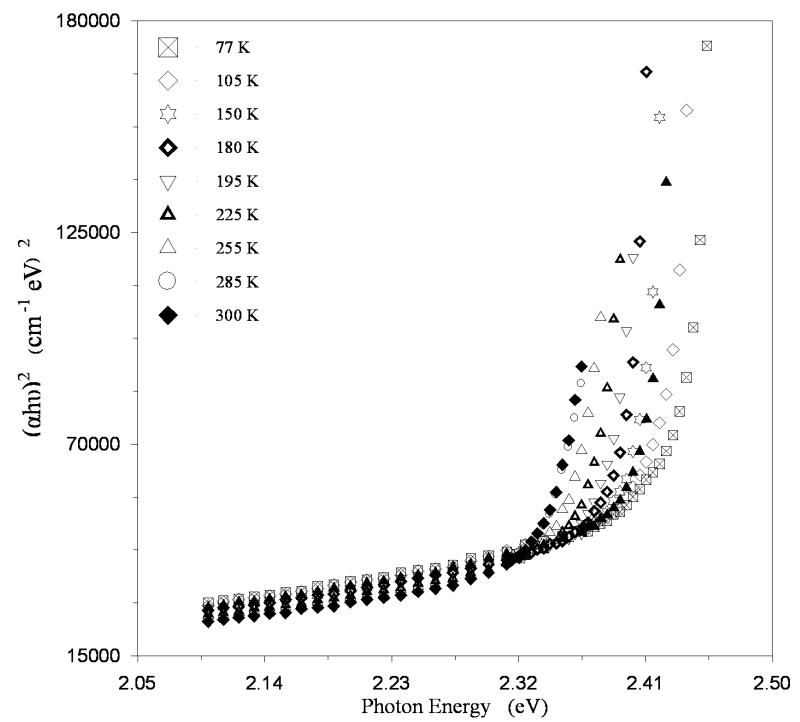

Fig. 9. The plot of $(\alpha h \nu)^{2}$ versus $h \nu$ for a thin film of $\mathrm{TlS}_{2}$.

of the thin films under investigation, the exponent $n$ indicates that the dominant transition is a direct allowed one. Therefore, $(\alpha h \nu)^{2}$ was plotted against $(h \nu)$ and this graph is described in Fig. 9. On the other hand, a usual method for determining the type of the optical transition includes plots of $(\alpha h \nu)^{1 / n}$ versus the incident photon energy $(h \nu)$. These proportionality gives a set of plots with four values of the exponent $n$ : $(\alpha h \nu)^{1 / 2}-h \nu$, of $(\alpha h \nu)^{2}-h \nu$, of $(\alpha h \nu)^{1 / 3}-h \nu$, and of $(\alpha h \nu)^{2 / 3}-h \nu$. One of these plots satisfies the widest linearity of data, and hence its exponent determines the type of the optical transition. In line with these plots, it was evident that the optical transition is a direct allowed one.

In this work, the optical band gaps were calculated by linear fitting the high absorption regions. These fits intersect the $h \nu$-axis at the values of the optical band gap widths of $\mathrm{TlS}_{2}$ thin films at the values of temperature under investigation. The figure also suggests a horizontal shift of the band edge (the increase in the motion of the atoms broadens the energy levels). As the temperature increases, the lattice expands and the oscillations of the atoms around their equilibrium lattice points increase. There is also an electron-lattice interaction, which depends strongly on temperature: at temperatures much lower than the Debye temperature, the gap width varies proportionally to the square of the temperature, whereas much above the Debye temperature the gap width varies linearly with the temperature [20]. But the temperature dependence of the band gap width for many semiconductors has been fitted by the following empirical relation:

$$
E_{\mathrm{g}}(T)=E_{\mathrm{g}}(0)-\left(\frac{\alpha T^{2}}{T+\beta}\right),
$$

where $E_{\mathrm{g}}(0)$ is the value of the energy gap at $0 \mathrm{~K}$, and $\alpha$ and $\beta$ are constants. The 


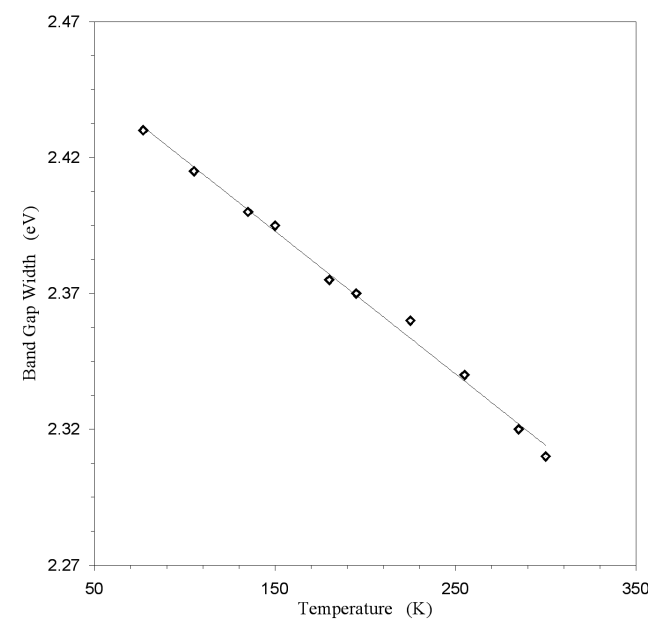

Fig. 10. The temperature dependence of the direct optical gap for a thin film of $\mathrm{TlS}_{2}$ as a result of the optical measurements.

temperature coefficient of the optical gap width may also be calculated using the slope of the linear fits for relation $E_{\mathrm{g}}$ versus $T$ (see Fig. 10). Using this figure, the value of $E_{\mathrm{g}}$ was calculated to be $\approx 2.31 \mathrm{eV}$ at room temperature, which increases with decreasing temperature to be $\approx 2.43 \mathrm{eV}$ at $77 \mathrm{~K}$. The temperature coefficient $\left(\mathrm{d} E_{\mathrm{g}} / \mathrm{d} T\right)$ was calculated to be $\approx-5.25 \times 10^{-4} \mathrm{eV} / \mathrm{K}$. The refractive indices $(n)$ over the range under investigation of temperature can be determined using the following relation:

$$
R=\frac{(n-1)^{2}+k^{2}}{(n+1)^{2}+k^{2}}
$$

But the thin film samples under investigation are transparent (the films are brown in color and transparent in appearance). Therefore, the above relation can be rewritten for the transparent range of irradiance in the form

$$
R=\left(\frac{n-1}{n+1}\right)^{2}
$$

Figure 11 shows the points that represent the calculated values of the refractive index in the low energy region of the studied incident photon energy range. It is evident from this figure that in the region mentioned in the investigated photon energy range, the dispersion of the refractive index is normal for all range of temperature under investigation and can be well described using a single oscillator model [23]:

$$
n^{2}=1+\frac{E_{0} E_{\mathrm{d}}}{E_{0}^{2}-(h \nu)^{2}} \Rightarrow\left(n^{2}-1\right)^{-1}=\frac{E_{0}}{E_{\mathrm{d}}}-\left(\frac{1}{E_{0} E_{\mathrm{d}}}\right)(h \nu)^{2},
$$

where $E_{0}$ is the single oscillator energy, $E_{\mathrm{d}}$ is the dispersion energy, and $h \nu$ is the incident photon energy. It is observed from Fig. 11 that the refractive indices 




Fig. 11. The photon energy dependence of the refractive index for a thin film of $\mathrm{TlS}_{2}$. Inset: the plot of $\left(n^{2}-1\right)^{-1}$ versus $(h \nu)^{2}$ for a thin film of $\mathrm{TlS}_{2}$.

TABLE

The optical and photoconductive parameters.

\begin{tabular}{c|c|c|c||c|c}
\hline \hline $\begin{array}{c}\text { Temp. } \\
{[\mathrm{K}]}\end{array}$ & $\begin{array}{c}\text { Optical gap }[\mathrm{eV}] \\
\text { (thin samples) }\end{array}$ & $\begin{array}{c}\text { Oscill. energy } \\
{[\mathrm{eV}]}\end{array}$ & $\begin{array}{c}\text { Disp. energy } \\
{[\mathrm{eV}]}\end{array}$ & \multicolumn{2}{|c}{$\begin{array}{c}\text { Energy gap of } \\
\text { bulk samples }\end{array}$} \\
\hline 77 & 2.430 & 3.73 & 5.40 & \multicolumn{2}{|c|}{$[\mathrm{eV}]$} \\
\cline { 5 - 6 } 105 & 2.415 & 3.60 & 4.99 & Temp. & Gap \\
\cline { 5 - 6 } 135 & 2.400 & 3.67 & 5.09 & 77 & 2.135 \\
150 & 2.395 & 4.02 & 6.00 & 110 & 2.073 \\
180 & 2.375 & 3.77 & 5.26 & 140 & 2.027 \\
195 & 2.370 & 3.53 & 4.56 & 180 & 1.977 \\
225 & 2.360 & 3.71 & 4.99 & 210 & 1.886 \\
255 & 2.340 & 3.47 & 4.26 & 250 & 1.807 \\
285 & 2.320 & 3.51 & 4.30 & 280 & 1.715 \\
300 & 2.310 & 3.34 & 3.78 & 300 & 1.649
\end{tabular}

for all studied temperature range vary linearly with the variation of the incident photon energy in the region of $2.1-2.2 \mathrm{eV}$. Both the single oscillator energy $\left(E_{0}\right)$ and the dispersion energy $\left(E_{\mathrm{d}}\right)$ can be obtained for all investigated temperatures by plotting $\left(n^{2}-1\right)^{-1}$ as a function of the photon energy $(h \nu)$ in the linear portion of the $n-\lambda$ plots $(2.1-2.2 \mathrm{eV})$, which is illustrated in the inset of Fig. 11. The $E_{\mathrm{d}}$ 
and $E_{0}$ were calculated from the slope $\left(E_{0} E_{\mathrm{d}}\right)^{-1}$ and the intercept $\left(E_{0} / E_{\mathrm{d}}\right)$, which is depicted in the inset of Fig. 11.

The static refractive index $n(0)=\left(1+E_{\mathrm{d}} / E_{0}\right)^{1 / 2}$ and the static dielectric constant $\left(\varepsilon_{\mathrm{s}}=n^{2}(0)\right)$ were calculated using the above reported relation and found to be $\approx 1.46$ and $\approx 2.13$, respectively. The calculated values of the oscillator energies $\left(E_{0}\right)$, the dispersion energies $\left(E_{\mathrm{d}}\right)$, and the optical gap widths $\left(E_{\mathrm{g}}\right)$ for the thin film samples are summarized in Table over the temperature range of $77-300 \mathrm{~K}$.

\section{Conclusion}

As a result of the above measurements, it can be concluded that the $\mathrm{TlS}_{2}$ is a good photoconductor. This can be observed in Fig. 2, which suggests that the photocurrent depends strongly on temperature from 77 to $300 \mathrm{~K}$. The above-mentioned dependence is an evidence that the $\mathrm{TlS}_{2}$ is considered as a photoconductor with high photosensitivity. The photopeak shifts toward low photon energy side with increasing temperature from 77 (it was found at $2.43 \mathrm{eV}$ ) to $300 \mathrm{~K}$ (it was found at $2.047 \mathrm{eV})$. The band gap width $\left(E_{\mathrm{g}}\right)$ of $\mathrm{Tl}_{2} \mathrm{~S}$ increases as temperature decreases from $300 \mathrm{~K}\left(E_{\mathrm{g}} \approx 1.65 \mathrm{eV}\right)$ to $77 \mathrm{~K}\left(E_{\mathrm{g}} \approx 2.14 \mathrm{eV}\right)$ with temperature coefficient $\mathrm{d} E_{\mathrm{g}} / \mathrm{d} T \approx-2.65 \times 10^{-3} \mathrm{eV} / \mathrm{K}$. Transmittance and reflectance spectra of the thin samples were measured in the temperature range of 77-300 K over the photon energy range of 2.1-2.45 eV. The absorption coefficient also exhibits a long band tail at low energies, which is most probably originated from defects and impurity states within the band gap. Tailing of the band states into the gap width may be induced from a large concentration of free carriers resulting from the screened Coulomb interaction between carriers that perturbs the band edges. The type of the optical transition was determined to be the direct allowed one. The direct allowed gaps were also determined to be $\approx 2.43 \mathrm{eV}$ at $77 \mathrm{~K}$ and $\approx 2.31 \mathrm{eV}$ at $300 \mathrm{~K}$, respectively. The temperature coefficient was calculated to be $-5.25 \times 10^{-4} \mathrm{eV} / \mathrm{K}$. The temperature dependence of refractive index was investigated. As results of this investigation, all of oscillator $E_{0}$, dispersion $E_{\mathrm{d}}$ energies of the refractive index were also evaluated from which both the static refractive index and the static dielectric constant were calculated.

\section{Acknowledgments}

The author is much indebted to Prof. Dr. A.E. Belal (Physics Department, Aswan Faculty of Science, South Valley University, Egypt) for his help and encouragement.

\section{References}

[1] A.E. Belal, A.T. Nagat, Cryst. Res. Technol. 25, K72 (1990).

[2] S. Kashida, K. Nakamura, J. Solid State Chem. 110, 264 (1994). 
[3] S. Kashida, T. Saito, M. Mori, Y. Tezuka, S. Shin, J. Phys., Condens. Matter 9, 46, 10271 (1997).

[4] A.S. Radtke, F.W. Dickson, Am. Mineral. 60, 559 (1975).

[5] J.A.A. Ketelaar, E.W. Gorter, Z. Kristallogr. 101, 367 (1939).

[6] L.I. Man, Sov. Phys. Crystallogr. 15, 399 (1970).

[7] G. Giester, C.L. Lengauer, E. Tillmanns, J. Zemann, J. Solid State Chem. 168, 322 (2002).

[8] H.A. Elshaikh, I.M. Ashraf, A.M. Badr, J. Phys. Chem. B 108, 11327 (2004).

[9] V. Estrella, M.T.S. Nair, P.K. Nair, Thin Solid Films 414, 289 (2002).

[10] I.M. Ashraf, H.A. Elshaikh, A.M. Badr, Phys. Status Solidi B 241, 885 (2004).

[11] I.M. Ashraf, H.A. Elshaikh, A.M. Badr, Cryst. Res. Technol. 39, 63 (2004).

[12] A.H. Wilson, Theory of Metals, 2nd ed., Cambridge University Press, Cambridge 1953.

[13] M. Soulard, M. Tournoux, Bull. Soc. Chim. Fr. 3, 791 (1971).

[14] T.S. Moss, Photoconductivity in Elements, Butterworths, London 1952.

[15] G. Busch, Phys. Acta. 19, 198, 32, Angew (1946).

[16] A.C. Rose, RCA Rev. 12, 362, 32 (1951).

[17] R.H. Bube, Photoconductivity of Solids, Wiley, New York 1960.

[18] S.H. Ryvkin, Photoelectric Effects in Semiconductors, Consultants Bureau, New York 1964.

[19] B. Gurbulak, Appl. Phys. A 58, 353 (1999).

[20] J.I. Pankove, Optical Processes in Semiconductors, Prentice-Hall, New Jersey 1971, p. 93.

[21] J. Tauc, Amorphous and Liquid Semiconductors, Plenum, New York 1974, Ch. 4.

[22] A.F. Qasrawi, Cryst. Res. Technol. 40, 610 (2005).

[23] C. Baban, G.I. Rusu, P. Prepelita, J. Optoelectron. Adv. Mater. 7, 817 (2005). 